This document is the Accepted Manuscript version of a Published Work that appeared in final form in Analytical Chemistry, copyright (c) American Chemical Society after peer review and technical editing by the publisher. To access the final edited and published work see:

https://dx.doi.org/10.1021/acs. analchem.9b03407. 


\title{
A smart chip for visual detection of bacteria using the electro- chromic properties of polyaniline
}

\author{
Saba Ranjbar ${ }^{\dagger, \star}$, Mohammad Amin Farahmand Nejad ${ }^{\dagger \dagger}$, Claudio Parolo ${ }^{\dagger}$, Saeed Shahrokhian ${ }^{\star}$, Arben \\ Merkoçi $i^{\dagger}, \| *$
}

† Nanobioelectronics and Biosensor Group, Catalan Institute of Nanoscience and Nanotechnology (ICN2), CSIC, The Barcelona Institute of Science and Technology, Campus UAB, Bellaterra, 08193, Barcelona, Spain

tDepartment of Chemistry, Sharif University of Technology, Tehran 11155-9516, Iran

// Catalan Institution for Research and Advanced Studies (ICREA), Pg. Lluís Companys 23, 08010 Barcelona, Spain

ABSTRACT: Finding fast and reliable ways to detect pathogenic bacteria is crucial for addressing serious public health issues in clinical, environmental and food settings. Here, we present a novel assay based on the conversion of an electrochemical signal into a more convenient optical read out for the visual detection of E. coli. Electropolymerizing polyaniline (PANI) on indium tin oxide screen printed electrode (ITO SPE), we achieved not only the desired electrochromic behavior, but also a convenient way to modify the electrode surface with antibodies (taking advantage of the many amine groups of PANI). Applying a constant potential to the PANI-modified ITO SPE induces a change in their oxidation state, which in turn generates a colour change on the electrode surface. The presence of E. Coli on the electrode surface increases the resistance in the circuit affecting the PANI oxidation states, producing a different electrochromic response. Using this electrochromic sensor, we could measure concentrations of E. Coli spanning four orders of magnitude with a limit of detection of $10^{2} \mathrm{CFU} \mathrm{mL} \mathrm{mL}^{-1}$. In this work we show that merging the sensitivity of electrochemistry with the user-friendliness of an optical read out can generate a new and powerful class of biosensors, with potentially unlimited applications in a variety of fields.

\section{Intorduction}

The highest number of annual hospitalizations and deaths within the United States are attributed to water resources contaminated with bacteria. ${ }^{1}$ Therefore, water monitoring for bacteria contamination is a number one priority for public health. With current technologies, testing for every single bacteria at the point of care is not feasible, that is why the United States Food and Drug Administration (FDA) suggested the use of the "generic" Escherichia Coli (E. Coli) level as an indicator for the overall bacterial contamination in fresh products and water samples. ${ }^{2}$ Current methods for the detection of E. Coli can be divided into laboratory-based tests (such as bacteria cultures and counting, ${ }^{3}$ enzyme-linked immunosorbent assay ${ }^{4}$ and polymerase chain reaction ${ }^{5}$ ) and point-of-care sensors ${ }^{6-8}$ (with the most common ones being lateral flow immunoassay - LFIA -9 and electrochemical sensors ${ }^{10}$ ). While the former methods provide high sensitivity and specificity they also require long, complicated, multi-step protocols (often involving expensive equipment) that prevent their use in applications where a fast detection is paramount. ${ }^{11}$ The latter instead are convenient to use at the point of care, but often times lack the sensitivity and specificity required by most applications (i.e. LFIA); or they require a portable equipment (potentiostat), which considerably increase their overall cost (i.e. electrochemical sensors). ${ }^{12-13}$

A method that combines the advantages of a simple optical read out and the portability of LFIA with the analytical capabilities of electrochemical sensors would provide a powerful tool for the accurate and ubiquitous analysis of fresh products and water samples. Electrochromic sensors ${ }^{14-15}$ provide exactly this: an optical readout that depends on the applied potential (which can be achieved with a device smaller and considerably cheaper than a fully portable potentiostat). ${ }^{16-17}$ This type of sensors harnesses the different colors that different oxidation states of electrochromic materials (ECMs). ${ }^{18}$ ECMs are classified into three categories including transition metal oxides (molybdenum, tungsten, titanium, iridium), single molecular materials (Prussian blue, Viologen) and $\pi$ conjugated polymer films (polyaniline, polypyrole, polythiophen) ${ }^{19-20}$ Considering the sensitivity of human eyes to the color change rather than color intensity, multicolor ECMs (such as $\pi$ conjugated polymer films and their nanocomposites) provide the most user friendly platform for point of care sensing. ${ }^{20-21}$ In this regard, polyaniline (PANI) is a unique ECM, having a fast response at closed applied potentials with a wide range of colors (yellow, green and blue)(Fig. 1). Besides its electrochromic behavior, PANI also provides high electrical conductivity, facile synthesis, high stability, simple (bio)functionalization (thanks to its - $\mathrm{NH} 2$ groups) and strong adhesion to the electrode surface, qualities that make it an ideal material for the development of point of care sensors. ${ }^{22-24}$

In the present study, we developed a novel electrochromic immunosensor based on the use of PANI and indium tin oxide (ITO) electrodes for the visual and fast detection of E.Coli in different water samples. 

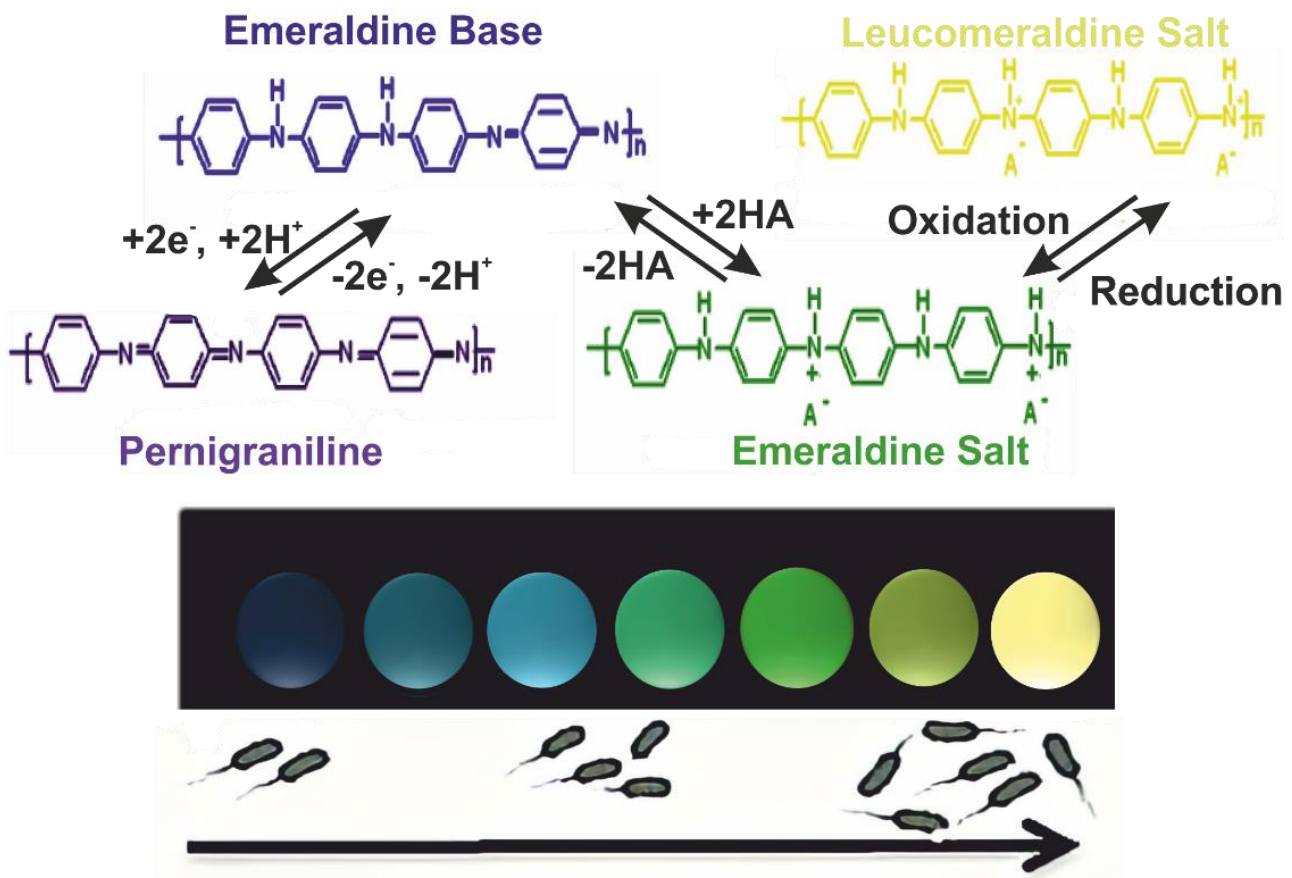

\section{E. Coli concentration}

Figure 1. Schematic illustration of different PANI oxidation states with their relative colors (penigraniline/blue-violet, emeraldine base/blue, emeraldine salt/green, leucoemeraldine salt/yellow) and how they can be related to different E. Coli concentrations.

\section{Experimental section}

\section{Chemical and Apparatus}

Aniline (99\%), sulphuric acid, potassium chloride, potassium hexacyanoferrate (III), potassium hexacyanoferrate (II), E. coli O157:H7 lyophilized cell, bovine serum albumin (BSA), glutaraldehyde (GA, 25\%), conductive indium tin oxide (ITO)/polyethylene terephthalate substrates (Rct $=60 \Omega \mathrm{cm}-1)$, ethanol, acetone, MES buffer and phosphate buffered saline tablets were obtained from Sigma-Aldrich (Madrid, Spain). Aniline was purified under vacuum distillation to obtain a colorless liquid and the other chemicals were used as received without further purification. Fresh Milli-Q water (Millipore) was used to prepare all aqueous solutions. Salmonella typhimurium (CECT 722T, S. typhimurium) strain was obtained from the Colección Española de Cultivos Tipo (CECT, Valencia, Spain).

Serial dilutions of bacteria were prepared in sterile fresh Milli-Q water and stored at $4{ }^{\circ} \mathrm{C}$ for one week. An E. coli serotype O/K Polyclonal antibody (PA1-7213, 4-5 mg/mL) was purchased from Thermo Fisher Scientific and diluted to 0.5 $\mathrm{mg} / \mathrm{mL}$ with sterile MES buffer (0.1M, $\mathrm{pH}=5.5)$.

DEK 248 screen-printing machine was used to prepare homemade screen-printed electrodes (SPEs) containing carbon ink as the counter electrode (CE), $\mathrm{AgCl}$ ink as the reference electrode (RE) and the insulating ink as a dielectric layer. The electrochemical experiments were performed by PGSTAT 302N controlled by a NOVA 1.11 software for voltammetric experiments and FRA software for impedimetric experiments. The optical study was conducted by applying different constant potentials to the PANI/ITO SPE, while absorption spectra were collected in 350-800 nm with Cary $4000 \mathrm{UV}-$ vis spectrophotometer.
All of the images were taken by a Samsung Galaxy J 5 pro, in manual mode, manual focus in ambient light conditions and images were analyzed with the ImageJ software.

Scanning electron microscopy (SEM) images were obtained using a FEI Quanta ${ }^{\mathrm{TM}} 650$ field emission gun scanning electron microscope (FEI, USA) to surface characterization of the electrode. XRD diagrams were collected on an X'Pert PRO MRD analytical diffractometer (Panalytical) at $45 \mathrm{kV}, 40 \mathrm{~mA}$ using $\mathrm{CuK} \alpha$ radiation $(\lambda=1.5406 \AA$ ). Fourier transform infrared (FTIR) spectra were recorded on a Bruker Tensor 27FTIR spectrometer equipped with a Golden Gate diamond attenuated total reflection (ATR) cell, in transmittance mode at room temperature. AFM experiments were carried out with a Veeco CP Research instrument using Si cantilever.

\section{ITO Screen-Printed Electrodes assembling}

The well-cleaned ITO slides $(1 \mathrm{~cm} \times 2.5 \mathrm{~cm})$ were obtained by pretreating slides in an ultrasonic bath for $15 \mathrm{~min}$ in each of the following solvents: soapy water, Milli-Q water, acetone, and ethanol sequentially. Then, the slides were rinsed with Milli-Q water and dried with a stream of compressed air. In following, the ITO SPEs were assembled by attachment of the ITO slides instead of the working electrode on the polyethylene terephthalate (PET) substrates containing counter electrode, a reference electrode, and dielectric layer by using double sided scotch tape.

\section{Immunosensor design and fabrication}

Prior to fabrication of electrochromic immunosensor, the ITO SPE was conditioned in PBS $(\mathrm{pH}=7.4,0.01 \mathrm{M})$ for 10 cycles using a potential range between $0.0 \mathrm{~V}$ to $1.0 \mathrm{~V}$ at the scan rate 
of $0.1 \mathrm{~V} \mathrm{~s}^{-1}$. The electrochromic thin film of polyaniline was obtained by drop casting of aniline monomer $(0.25 \mathrm{M}$ in $0.5 \mathrm{M}$ $\left.\mathrm{H}_{2} \mathrm{SO}_{4}\right)$ at the surface of ITO SPE and electropolymerization of aniline with cyclic voltammetry $(\mathrm{CV})$ technique in the potential range of $-0.8 \mathrm{~V}$ to $1.0 \mathrm{~V}$ under the scan rate of $0.1 \mathrm{~V} \mathrm{~s}^{-1}$ during 6 cycle (optimum conditions). To improve the sensitivity of the detection system, the leuocoemeraldine form of polyaniline (yellow color) was stabilized by casting $50 \mu \mathrm{L}$ of $\mathrm{H}_{2} \mathrm{SO}_{4}(0.5$ $\mathrm{M})$ at the surface of the electrode and applying the fixed potential of $-0.4 \mathrm{~V}$ during $50 \mathrm{~s}$. Afterward, the antibody was covalently conjugated to the PANI using glutaraldehyde (GA) as a cross-linking agent. For this purpose, after washing the electrode with Milli-Q water carefully, the $5 \mu \mathrm{L}$ of GA $(0.25 \%)$ was dropped at the surface of PANI/ITO SPE to activate the amine groups of polyaniline for $30 \mathrm{~min}$ followed by rinsing with Milli$\mathrm{Q}$ water to remove unbound GA. Next, $10 \mu \mathrm{L}$ of anti-E. coli antibody $(0.5 \mathrm{mg} / \mathrm{mL})$ was incubated at the surface of GA/PANI/ITO SPEs and kept $2 \mathrm{~h}$ in room temperature, then the electrode was blocked using $5 \mu \mathrm{L}$ of $1 \%$ BSA casted at the surface of anti- $E$. coli/GA/PANI/ITO SPE for $30 \mathrm{~min}$ to minimize the nonspecific binding which then rinsed with Milli-Q water again to remove unbounded BSA. Finally, the prepared immunosensor was immersed in different concentration of $E$. coli for $60 \mathrm{~min}$ for antigen-antibody bonding.
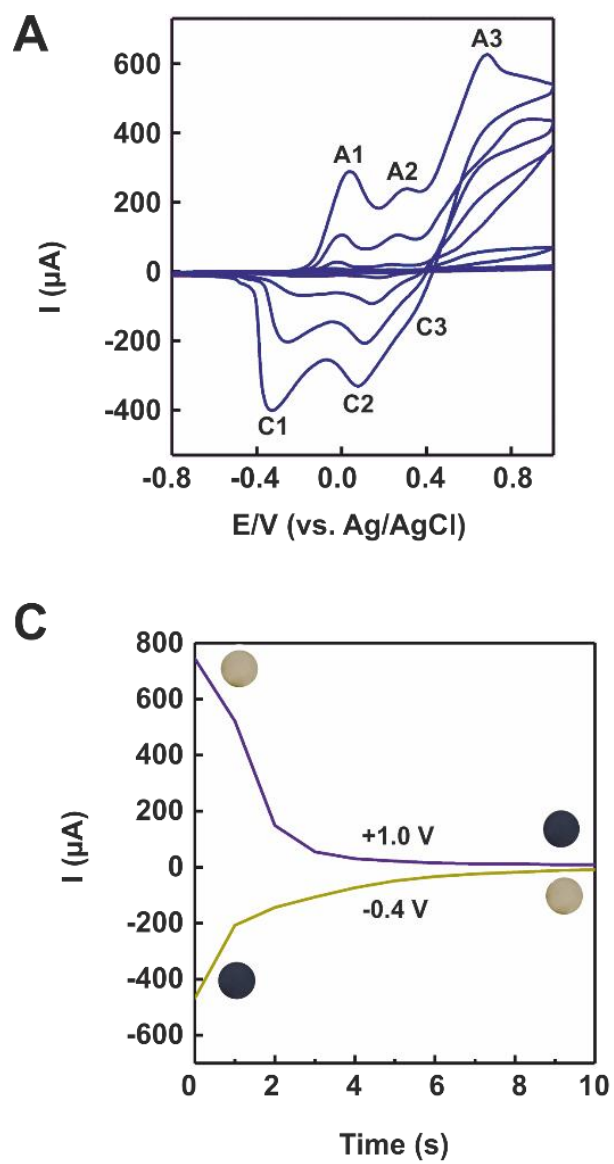

\section{E. coli 0157:H7 detection}

The visual detection of $E$. coli $\mathrm{O} 157: \mathrm{H} 7$ was conducted by applying constant potential $(0.2 \mathrm{~V})$ to the electrochromic immunosensor and following the PANI color changes in the presence the different concentration of $E$. coli $\mathrm{O} 157: \mathrm{H} 7$. Due to steric hindrance occurred on the surface of PANI/ITO SPE by forming the antibody-antigen complex, the color change of working electrode disrupted during apply constant potential. The polyaniline color changes in wide color range from yellow to blue were followed by increasing the concentration of $E$. coli O157:H7 by the naked eye and smartphone and also analyzed with ImageJ software to the detection of E. coli O157:H7.

\section{Real sample preparation}

Applicability of the designed electrochromic immunosensing platform was confirmed by testing of electrodes in different water samples. These experiments were conducted by spiking $10^{2}$

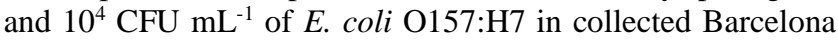
tap water and Ter river water (three replicates for each bacterial concentration in each sample).
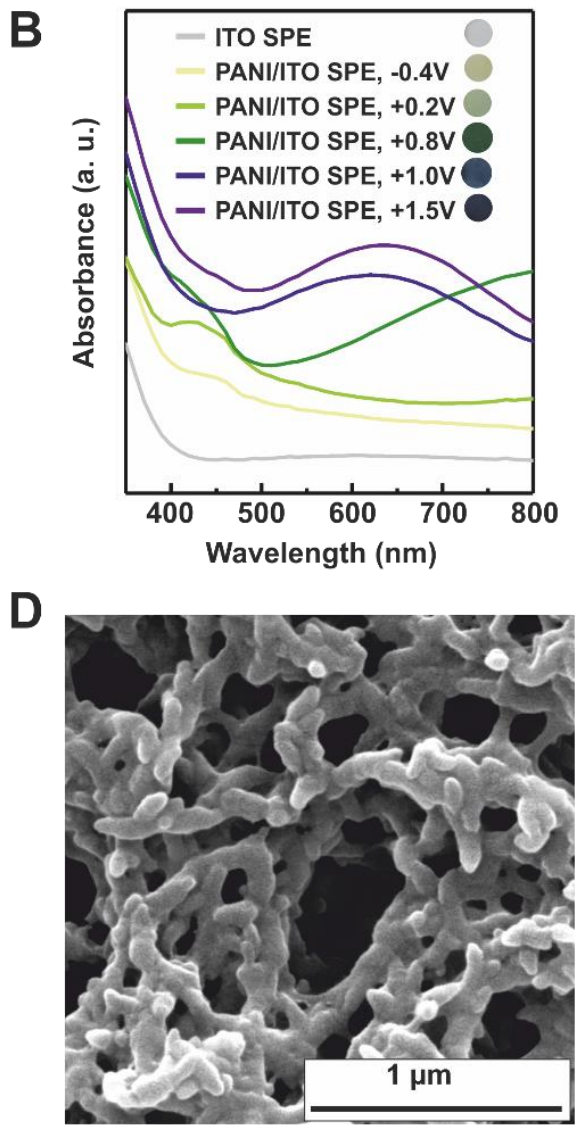

Figure 2: Characterization of PANI/ITO screen printed electrodes. (A) Cyclic voltamograms showing the formation of the thin film of PANI. The three redox couples $\mathrm{A} 1 / \mathrm{C} 1, \mathrm{~A} 2 / \mathrm{C} 2$, and $\mathrm{A} 3 / \mathrm{C} 3$ correspond respectively to: lucoemeraldine base, emeraldine salt and emeraldine base. (B) The diferent absorbance spectra further confirm the formation of the thin film of PANI. In the basnece of PANI, ITO SPEs do not show any peak, indicating their transparency, while the application of different potential to the PANI thin film produces characteristic peaks: the one at 450 represent the yellow color of lucoemeraldine base and the one at $650 \mathrm{~nm}$ represents emeraldine. (C) Chronoamperogram showing the change in oxidation and reduction state over time applying a fixed potential. (D) FESEM image showing the fibrous morphology of the PANI thin film. 


\section{Results and discussion}

The thin film of polyaniline (PANI) was obtained on the ITO SPE with cyclic voltammetry (CV) technique using a solution containing $0.25 \mathrm{M}$ aniline monomer in $0.5 \mathrm{M} \mathrm{H}_{2} \mathrm{SO}_{4}$ during 6 cycles. By scanning the potential from $-0.8 \mathrm{~V}$ to $1.0 \mathrm{~V}$ during electropolymerization of aniline on the ITO SPE (Fig. 2A), we observe three peaks (corresponding to three redox couples $\mathrm{A} 1 / \mathrm{C} 1, \mathrm{~A} 2 / \mathrm{C} 2$, and $\mathrm{A} 3 / \mathrm{C} 3$ ) of three different aniline oxidation states: lucoemeraldine base (LB, fully reduced) to emeraldine salt (ES, partially oxidized) and to emeraldine base (EB, fully oxidized).19 Thanks to its electrochromic properties, we can follow the PANI film electropolymerization directly on the working electrode surface, which changes color from pale yellow to green and blue by scanning to more positive potentials, while it changes from blue to pale yellow toward a negative direction (movie 1, clip of PANI film formation in S.I.).

The main reason why we decided to use ITO electrodes is because of their transparency, which greatly facilitates the different colors recognition (besides providing good electrical conductivity and a cheap and easy manufacturing). As expected ITO electrodes without PANI is fully transparent, thus failing in producing any absorption peak, while different oxidation states of the PANI film formation produced specific absorption spectra. The bands at $450 \mathrm{~nm}$ is related to $\pi-\pi^{*}$ transition of benzene rings, characteristic of LB form from yellow to yellowish green in the potential range of $-0.4 \mathrm{~V}$ to $0.2 \mathrm{~V}$. As the applied potential increased, this characteristic peak disappeared and the new one observed at $650 \mathrm{~nm}$, demonstrate formation of emeraldine which contains conjugated rings. ${ }^{25}$ The coloration switching response of PANI is followed by recording chronoamperogram of PANI/ITO SPE in $0.5 \mathrm{M} \mathrm{H}_{2} \mathrm{SO}_{4}$ during a redox reaction. Fig. $2 \mathrm{C}$ reports the current-time events by applying $0.4 \mathrm{~V}$ (movie 2. clip of reduction reaction in S.I.) and $+1.0 \mathrm{~V}$ (movie 3. clip of oxidation reaction in S.I.). The time of switching between fully reduced (LB) and fully oxidized (EB) is found to be $1.25 \mathrm{~s}$, meaning that the average switching time between three PANI redox states (LB to ES to EB) is approximately $0.4 \mathrm{~s}$, which is suitable for the fabrication of the fast multicolor electrochromic device.

To further confirm the electropolymerization of aniline at the surface of the working electrode, we characterized the resulting ITO SPE using Field Emission Scanning Electron Microscope (FESEM), Energy-dispersive X-ray spectroscopy (EDS), X-ray powder diffraction (XRD), and Fourier-transform infrared spectroscopy (FTIR) analysis. Fig. 2D shows the fibrous morphology for PANI with a uniform distribution of chains over the surface of ITO SPE, which were further confirmed by detecting carbon, nitrogen, and sulphur in EDS data (Fig. S1 A) and by the FTIR spectra (Fig. S1 B). In particular, the well-resolved peaks at 1495 and $1575 \mathrm{~cm}^{-1}$ corresponded to the $\mathrm{C}=\mathrm{C}$ stretching vibration of benzenoid and quinonoid rings, the peak at $1304 \mathrm{~cm}^{-1}$ corresponds to the $\mathrm{C}-\mathrm{N}$ stretching vibration with aromatic conjugation, the peak observed at $1153 \mathrm{~cm}^{-1}$ corresponds to $\mathrm{Q}=\mathrm{NH}+\mathrm{B}$ stretching $(\mathrm{Q}$ and $\mathrm{B}$ are quinonoid and benzene rings respectively) and, especially, the peak between 2500-3500 $\mathrm{cm}^{-1}$ corresponds to the $=\mathrm{N}-\mathrm{H}$ stretching vibration. ${ }^{25} \mathrm{We}$ also studied the polymer crystal structure by XRD (Fig. S1 C), whose data show peaks at $2 \theta=22^{\circ}$ and $2 \theta=26^{\circ}$ that are originated from (020) and (200) crystalline phase of PANI. As compared with the highly crystalline polyethylene terephthalate (PET) of bare ITO SPE, the peaks of PANI are relatively small and merged with PET peaks (however, for better illustration of
PANI peaks the XRD spectrum of PANI obtained by the same procedure on SPE and shown in Fig. S2 C). ${ }^{26}$

In order to extend the applications of polyaniline as an electrochromic material for using in biosensing platform, the electrochromic feature of PANI was evaluated in phosphate buffer saline (PBS, 0.1 M, pH = 7.4) as a biological electrolyte. ${ }^{27} \mathrm{Be}-$ ing electrochromic properties $\mathrm{pH}$ and saline dependent, it is no surprise that the PANI behavior in PBS differs from the one in $0.5 \mathrm{M} \mathrm{H}_{2} \mathrm{SO}_{4}$. In particular, we observed that the LB form of PANI is less stable in PBS than in $\mathrm{H}_{2} \mathrm{SO}_{4}$, thus requiring a lower potential to switch to the EB form (Fig. S2). The effect of the higher $\mathrm{pH}$ of PBS, also speed up the conversion of LB to ES and $\mathrm{EB}$ form, which would also happen at lower potentials. We believe that this different behavior is due to the deprotonation of aniline ring in high $\mathrm{pH}$ values. ${ }^{28}$

As the PANI shows electrochromic behavior just when in the thin film conformation, we produced different electrodes with different thicknesses, obtained by changing the number of $\mathrm{CV}$ cycles used for the electropolymerization. We found that the electrochromic behavior of PANI appears on the fabricated ITO SPE after $4 \mathrm{CV}$ cycles, but it reaches the appropriate stability and wide color range at 6 cycles. Increasing the number of cycles over 6 decreases the color range, since it stabilizes the emeraldine form impeding the formation of the lucoemeraldine (yellow color). ${ }^{28}$ Once optimized the thin film fabrication for our purpose, we characterized it using Atomic force microscopy (AFM) (Fig. S3), finding that the final thickness, using the 6 cycles CV deposition, is around $36.4 \pm 4.4 \mathrm{~nm}$. The nm-size thickness causes a considerable increase in the active surface area for the electrode to improve the immobilization of antibody.

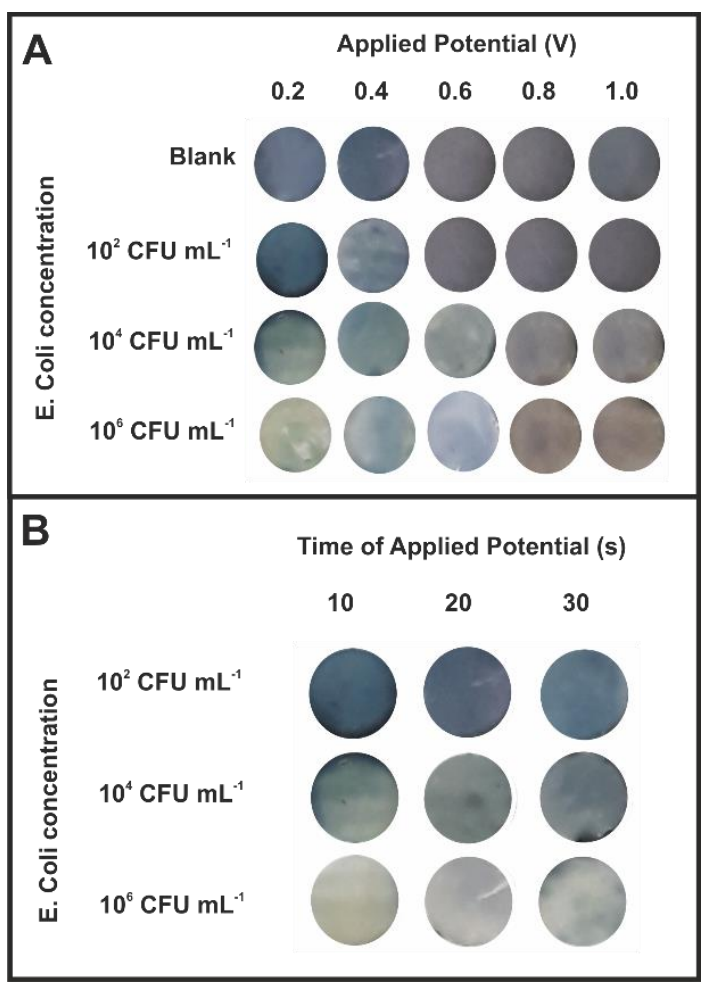

Figure 3. Effect of the applied potential (A) and its application time (B) to the signal generation for different E. Coli concentrations. The use of high potentials limits the response range (we selected $0.2 \mathrm{~V}$ as the optimal parameter). Instead the time does not affect much the response (we thus selected $10 \mathrm{sec}$ ) 
The amount of antibody immobilized on the surface of the electrode is a key factor to efficiently capture the analyte. Thus, we tested three different amounts of antibody (using solutions with $2.5,5.0$ and $10.0 \mu \mathrm{g} / \mathrm{mL}$ of antibody), which we immobilized using glutaraldehyde as cross-linking agent. We found that the smallest concentration failed in providing enough binding capability to accumulate enough bacteria to affect the electrochromic properties of PANI (the color was always dark blue for all concentration of E. coli O157:H7 we tested). Instead, the highest concentration was so high that the antibody itself was affecting PANI electrochromic behavior, saturating the system even before the capture of the bacteria. The middle concentration was the only one that provided enough binding capability without affecting the sensor before the binding of the bacteria happens. It provided the full color range from yellow to green and blue. These results are depicted in Fig. S4 A. The interaction time between E. coli and anti-E. coli antibody was optimized using a concentration of $10^{4} \mathrm{CFU} / \mathrm{mL}$ of $\mathrm{E}$. coli. We found that the optimal incubation time to observe a color change corresponded to $60 \mathrm{~min}$ (Fig. S4 B).

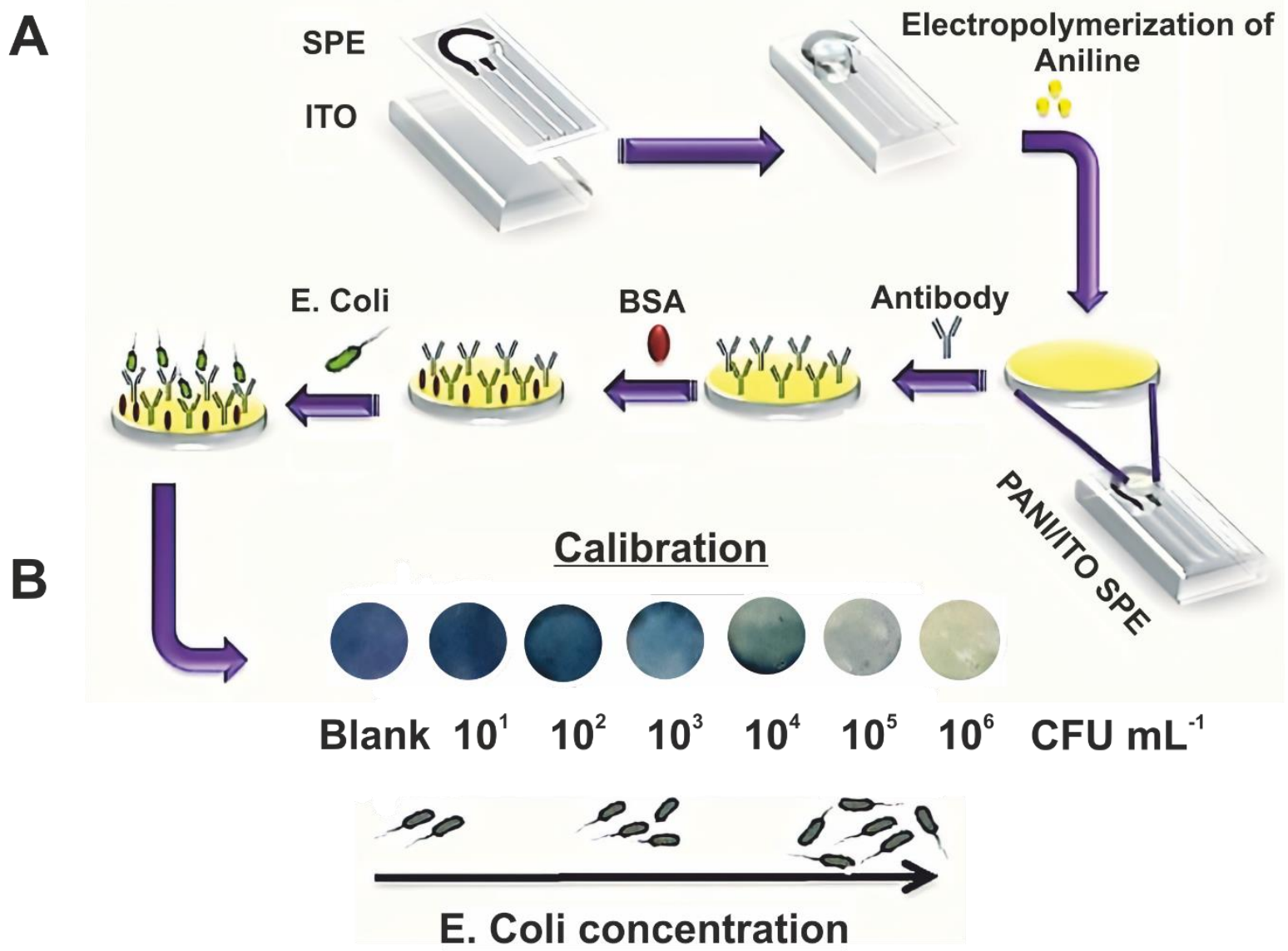

C

Selectivity

$10^{6} \mathrm{CFU} \mathrm{mL} \mathrm{m}^{-1}$ of E.coli

$10^{6} \mathrm{CFU} \mathrm{mL} \mathrm{m}^{-1}$ of S. typhymurium

$5 \times 10^{5} \mathrm{CFU} \mathrm{mL} \mathrm{m}^{-1}$ of E.coli +

$5 \times 10^{5} \mathrm{CFU} \mathrm{mL}^{-1} \mathrm{~S}$. typhymurium
D Real Samples

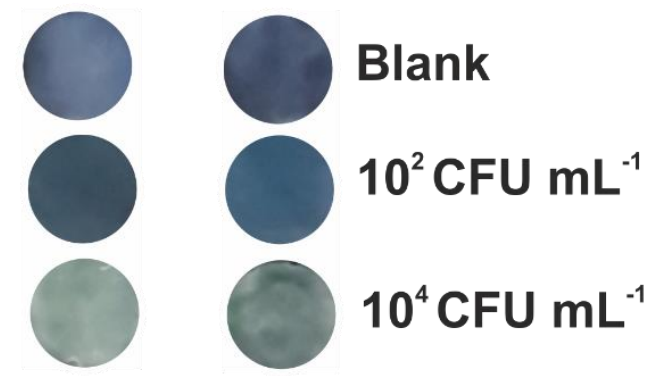

\section{Tap River water water}

Figure 4: Schematic and performance of the proposed electrochromic sensor. A) Scheme of sensor fabrication and utilization. B) Sensor output for different concentrations of E. Coli. C) Sensor selectivity using S. typhymorium as interferent. D) Real samples results using tap water and Ter river water spiked with E. Coli. 
One of the most important parameters in an electrochromic sensor is the choice of the applied potential, since it defines the dynamic range of the sensor and its sensitivity. In our case we tested one blank and three different target concentrations $\left(10^{2}\right.$, $\left.10^{4}, 10^{6} \mathrm{CFU} / \mathrm{mL}\right)$ vs five different potentials $(0.2$. 0.4, 0.6, 0.8, $1 \mathrm{~V}$ ). The results (Fig. 3A) show how the smallest potential applied $(0.2 \mathrm{~V})$ was the one that provided the highest sensitivity and color change, allowing the discrimination of the three different E. coli concentrations. Using higher potential lead to a minor sensitivity due to the fact the complex antibody/bacteria was not enough to disrupt the PANI electrochromic properties. We also tested different times for the applied potential (Fig. 3B), but this parameter did not affect much the system, thus we decided to use the shortest time of $10 \mathrm{sec}$.

Having an insight into the fabrication and detection process can be very handy when preparing a new biosensing device (i.e. checking for potential issues before running the sample can save both time and money, besides it can confirm what happens on the electrode surface). Taking advantage of the electrochemical capabilities of our system we managed to do just that, using cyclic voltammetry $(\mathrm{CV})$ and electrochemical impedance spectroscopy (EIS) methods in the presence of redox probe $(0.1 \mathrm{M}$ $\mathrm{KCl}$ containing $\left.1 \mathrm{mM} \mathrm{Fe}(\mathrm{CN})_{6}^{3-/ 4-}\right)$. First we confirmed the formation of PANI thin film onto the SPE electrode surface. Thanks to the high conductivity and surface area of PANI, once the thin film is produced we observed both an increase in the peak current as well as a decrease in the charge transfer resistance (Rct). Then we verified the correct surface functionalization with anti-E. Coli antibody observing a decrease in the peak current and an increase in the Rct. This behavior is due to the steric hindrance that antibodies produce on the electrode surface, limiting in fact the contact between the electrode and the redox probe. Similarly, the addition of E. Coli produced a further peak decrease and increased Rct, due to an even higher steric hindrance effect produce by the bacteria on the surface (Fig. S5).

After the optimization and characterization of our electrochromic sensor, we proceeded to evaluate its analytical performances for the detection of $E$. Coli. First we challenged it with concentrations of $E$ Coli from $10^{1}$ to $10^{6} \mathrm{CFU} \mathrm{ml}^{-1}$, showing that

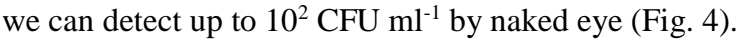

Then we tested the reproducibility of the results using five different electrodes and challenging them with concentration of E. Coli of $10^{2}$ and $10^{6} \mathrm{CFU} \mathrm{ml}^{-1}$, obtaining respectively relative standard deviation of $5.65 \%$ and $4.39 \%$ as proof of the high reproducibility of our sensing platform. We also proved its specificity challenging it with $10^{6} \mathrm{CFU} \mathrm{m}{ }^{-1}$ of $S$. typhymurium as well as a mix of E. coli and S. typhymurium. The sensor showed an excellent specificity changing color just for the sample with salmonella, indicating that the antibodies did not recognized it as a target (Fig. 4). Finally, we decided to use our test for the detection of $E$. Coli in real samples using tap water from Barcelona and water from the Ter river (Spain). Both water samples came out clean, so we decided to spike them with $10^{2}$ and $10^{4}$ CFU mL $\mathrm{mL}^{-1}$ of . Coli. In both case we succeed to detect the presence of the spiked $E$. Coli, demonstrating that our sensor could provide a novel tool for the analysis of bacterial contaminant in water samples (Fig. 4).

\section{Conclusion}

In summary, here we presented a novel electrochromic sensor for the convenient optical detection of bacteria in water sample at the point of care. The outstanding analytical performances in terms of sensitivity, specificity and ease of use make of this sensor a great tool for environmental and food-safety applications, that go beyond the detection of E. Coli.

\section{ASSOCIATED CONTENT}

\section{Supporting Information}

Supporting information includes: EDS, XRD and FTIR analysis of synthesized PANI; The absorbance spectra obtained for PANI in PBS; The AFM images of PANI thin film; Optimization of anti E. coli antibody amount and E. coli incubation time; Cyclic voltammograms and Nyquist plots in each steps of immunosensor fabrication.

The Supporting Information is available free of charge on the ACS Publications website.

\section{AUTHOR INFORMATION}

\author{
Corresponding Author \\ *Arben Merkoçi, Tel.: +34 937374604 \\ E-mail address: arben.merkoci@icn2.cat.
}

\section{Author Contributions}

The manuscript was written through contributions of all authors. / All authors have given approval to the final version of the manuscript. /

Notes

Any additional relevant notes should be placed here.

\section{ACKNOWLEDGMENT}

The ICN2 is funded by the CERCA Programme / Generalitat de Catalunya. The ICN2 is supported by the Severo Ochoa program of the Spanish Ministry of Economy, Industry and Competitiveness (MINECO, grant No. SEV-2017-0706 and MAT2017-87202-P). C.P. acknowledges Marie Skłodowska-Curie Actions Individual Fellowship (Funding scheme: MSCA-IF-EF-RI, Proposal number: 795635). The Iranian authors gratefully acknowledge the Iranian Ministry of Science, Research and Technology (MSRT) to provided sabbatical leave.

\section{REFERENCES}

(1) Kim, S. U.; Jo, E.-J.; Mun, H.; Noh, Y.; Kim, M.-G. Ultrasensitive Detection of Escherichia coli O157: H7 by Immunomagnetic Separation and Selective Filtration with Nitroblue Tetrazolium/5-Bromo4-chloro-3-indolyl Phosphate Signal Amplification. J. Agric. Food Chem. 2018, 66 (19), 4941-4947.

(2) Wang, D.; Chen, J.; Nugen, S. R. Electrochemical detection of Escherichia Coli from aqueous samples using engineered phages. Anal. Chemistry 2017, 89 (3), 1650-1657.

(3) Fukushima, H.; Katsube, K.; Hata, Y.; Kishi, R.; Fujiwara, S. Rapid separation and concentration of food-borne pathogens in food samples prior to quantification by viable-cell counting and real-time PCR. Appl. Environ. Microbiol. 2007, 73 (1), 92-100.

(4) Guo, Q.; Han, J.-J.; Shan, S.; Liu, D.-F.; Wu, S.-S.; Xiong, Y. H.; Lai, W.-H. DNA-based hybridization chain reaction and biotinstreptavidin signal amplification for sensitive detection of Escherichia coli O157: H7 through ELISA. Biosens. Bioelectron. 2016, 86, 990995.

(5) Bian, X.; Jing, F.; Li, G.; Fan, X.; Jia, C.; Zhou, H.; Jin, Q.; Zhao, J. A microfluidic droplet digital PCR for simultaneous detection of pathogenic Escherichia coli O157 and Listeria monocytogenes. Biosens. Bioelectron. 2015, 74, 770-777. 
(6) Cheeveewattanagul, N.; Morales-Narváez, E.; Hassan, A. R. H.; Bergua, J. F.; Surareungchai, W.; Somasundrum, M.; Merkoçi, A. Straightforward immunosensing platform based on graphene oxidedecorated nanopaper: a highly sensitive and fast biosensing approach. Adv.d Funct. Mater. 2017, 27 (38), 1702741.

(7) Peng, H.; Chen, I. A. Rapid Colorimetric Detection of Bacterial Species through the Capture of Gold Nanoparticles by Chimeric Phages. ACS nano 2019.

(8) Sadat Ebrahimi, M.-M.; Voss, Y.; Schönherr, H. Rapid detection of Escherichia coli via enzymatically triggered reactions in self-reporting chitosan hydrogels. ACS Appl. Mater. Interfaces 2015, 7 (36), 20190-20199.

(9) Morales-Narváez, E.; Naghdi, T.; Zor, E.; Merkoçi, A. Photoluminescent lateral-flow immunoassay revealed by graphene oxide: highly sensitive paper-based pathogen detection. Anal. Chem. 2015, 87 (16), 8573-8577.

(10) Luo, Z.; Yang, D.; Qi, G.; Yuwen, L.; Zhang, Y.; Weng, L.; Wang, L.; Huang, W. Preparation of highly dispersed reduced graphene oxide decorated with chitosan oligosaccharide as electrode material for enhancing the direct electron transfer of Escherichia coli. ACS Appl. Mater. interfaces 2015, 7 (16), 8539-8544.

(11) Amiri, M.; Bezaatpour, A.; Jafari, H.; Boukherroub, R.; Szunerits, S. Electrochemical methodologies for the detection of pathogens. ACS sensors 2018, 3 (6), 1069-1086.

(12) Kuss, S.; Couto, R. A. d. S.; Evans, R. M.; Lavender, H.; Tang, C.; Compton, R. G. Versatile Electrochemical Sensing Platform for Bacteria. Anal. Chem. 2019.

(13) Gao, G.; Wang, D.; Brocenschi, R.; Zhi, J.; Mirkin, M. V. Toward the Detection and Identification of Single Bacteria by Electrochemical Collision Technique. Anal. Chem. 2018, 90 (20), 12123 12130.

(14) Capoferri, D.; Álvarez-Diduk, R.; Del Carlo, M.; Compagnone, D.; Merkoçi, A. Electrochromic molecular imprinting sensor for visual and smartphone-based detections. Anal. Chem. 2018, 90 (9), 58505856.

(15) Cheng, W.; Ju, Y.; Payamyar, P.; Primc, D.; Rao, J.; Willa, C.; Koziej, D.; Niederberger, M. Large-Area Alignment of Tungsten Oxide Nanowires over Flat and Patterned Substrates for Room-Temperature Gas Sensing. Angew. Chem. Int. Ed. 2015, 54 (1), 340-344.

(16) Xu, W.; Fu, K.; Bohn, P. W. Electrochromic sensor for multiplex detection of metabolites enabled by closed bipolar electrode coupling. ACS sensors 2017, 2 (7), 1020-1026.

(17) Zhai, Q.; Zhang, X.; Xia, Y.; Li, J.; Wang, E. Electrochromic sensing platform based on steric hindrance effects for CEA detection. Analyst 2016, 141 (13), 3985-3988.
(18) Yu, Z.; Cai, G.; Ren, R.; Tang, D. A new enzyme immunoassay for alpha-fetoprotein in a separate setup coupling an aluminium/Prussian blue-based self-powered electrochromic display with a digital multimeter readout. Analyst 2018, 143 (13), 2992-2996.

(19) Zhang, J.; Tu, J.-p.; Zhang, D.; Qiao, Y.-q.; Xia, X.-h.; Wang, X.-1.; Gu, C.-d. Multicolor electrochromic polyaniline-WO3 hybrid thin films: one-pot molecular assembling synthesis. J. Mater. Chem. 2011, 21 (43), 17316-17324.

(20) Alesanco, Y.; Viñuales, A.; Palenzuela, J. s.; Odriozola, I.; Cabañero, G. n.; Rodriguez, J.; Tena-Zaera, R. n. Multicolor electrochromics: rainbow-like devices. ACS Appl. Mater. Interfaces 2016, 8 (23), 14795-14801

(21) Jensen, J.; Hösel, M.; Dyer, A. L.; Krebs, F. C. Development and Manufacture of Polymer-Based Electrochromic Devices. Adv. Funct. Mater. 2015, 25 (14), 2073-2090.

(22) Hu, F.; Xu, J.; Zhang, S.; Jiang, J.; Yan, B.; Gu, Y.; Jiang, M.; Lin, S.; Chen, S. Core/shell structured halloysite/polyaniline nanotubes with enhanced electrochromic properties. J. Mater. Chem. C 2018, 6 (21), 5707-5715

(23) Shahrokhian, S.; Ranjbar, S. Aptamer immobilization on amino-functionalized metal-organic frameworks: an ultrasensitive platform for the electrochemical diagnostic of Escherichia coli O157: H7. Analyst 2018, 143 (13), 3191-3201.

(24) Zhang, S.; Sun, G.; He, Y.; Fu, R.; Gu, Y.; Chen, S. Preparation, characterization, and electrochromic properties of nanocellulose-based polyaniline nanocomposite films. ACS Appl. Mater. Interfaces 2017, 9 (19), 16426-16434.

(25) Porcel-Valenzuela, M.; Ballesta-Claver, J.; de Orbe-Payá, I.; Montilla, F.; Capitán-Vallvey, L. F. Disposable electrochromic polyaniline sensor based on a redox response using a conventional camera: A first approach to handheld analysis. J. Electroanal. Chem. 2015, 738, 162-169.

(26) Bera, A.; Deb, K.; Kathirvel, V.; Bera, T.; Thapa, R.; Saha, B. Flexible diode of polyaniline/ITO heterojunction on PET substrate. Appl. Surf. Sci. 2017, 418, 264-269.

(27) Zhou, K.; Wang, H.; Jiu, J.; Liu, J.; Yan, H.; Suganuma, K. Polyaniline films with modified nanostructure for bifunctional flexible multicolor electrochromic and supercapacitor applications. Chem. Eng. J.1 2018, 345, 290-299.

(28) Marmisollé, W. A.; Florit, M. I.; Posadas, D. Coupling between proton binding and redox potential in electrochemically active macromolecules. The example of Polyaniline. J.Electroanal. Chem. 2013, $707,43-51$

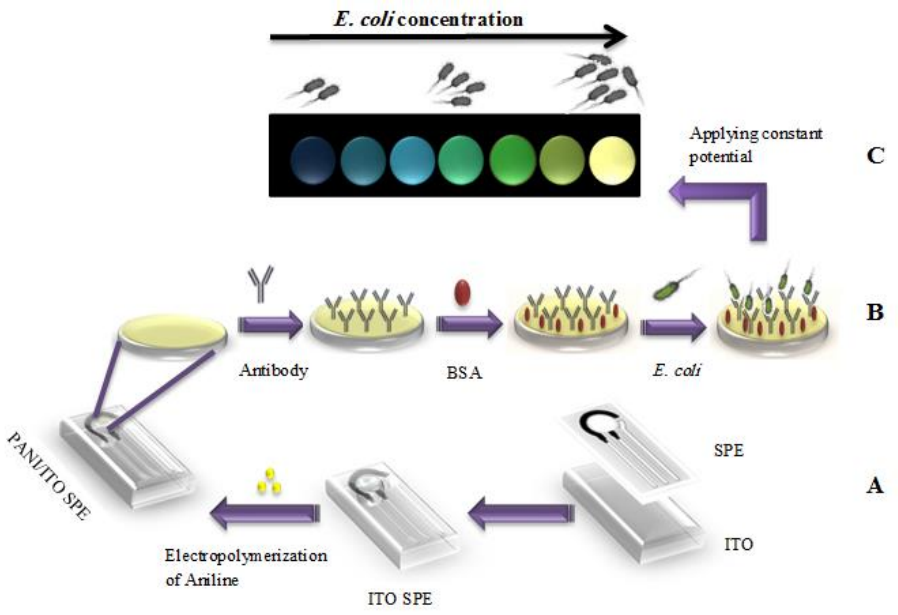

